\title{
HAUTE COUTURE, HAUTE CUISINE, HAUTE GAMES
}

\author{
Maggie Parker \\ School of Computing \\ University of Teesside \\ Borough Road \\ Middlesbrough. UK \\ m.parker@tees.ac.uk
}

\author{
Clive Fencott \\ School of Computing \\ University of Teesside \\ Borough Road \\ Middlesbrough, UK \\ p.c.fencott@tees.ac.uk
}

\author{
Paul van-Schaik \\ School of Social Sciences and \\ Law \\ University of Teesside \\ Borough Road \\ Middlesbrough, UK \\ P.Van-Schaik@tees.ac.uk
}

\begin{abstract}
Computer games provide a unique opportunity to experience intangible fantasies and create engagement with emotions which are able to be played out in real-life. Artists speak of engagement, computer game players speak of transformation and immersion. They are all viewing images with their own personal narratives informing their participation. These personal narratives arise from the information provided by the participant's self and self-identity. Artists and game players are modding games to suit their own purposes, creating new types of 'Haute Games' in a similar style to 'Haute Cuisine' or 'Haute Couture'. The importance of alternative computer-game genres which allow personal blendings for game players has been investigated and identified.
\end{abstract}

\section{INTRODUCTION}

All ideas must stem from somewhere and fine artists have the passion of their work to generate ideas. Game developers speak constantly of how they love playing games and also how they love creating them. In the creative industries, passion and love are spoken of often. However, for many people computer games $=$ youth and violence These people who could become game players felt excluded from playing games. This raised the question of what other methods could be used to interact with game-players. When playing computer games, players can command armies, rule worlds, and change identities. Players can navigate through unfamiliar territory, shoot, maim and kill 'people', build cities and solve puzzles. They can change identities, for example when playing 'Sims' or 'Fable' or 'World of Warcraft' players can become alternative genders or creatures inhabiting these virtual worlds, but games can be used to do and create other meanings.

\section{Haute games}

The following games discussed in this section are games that have been categorised as 'Haute Games'. These games are the ones that 'take risks' These games can investigate, highlight and create new and alternative game genres by taking risks, which is something artists know intimately and live with every day. In art every piece of artwork placed for public viewing is a risk, for example the risk of exposing something the artist wishes to conceal-revealing a part of the artists' self, for example when making a self-portrait. There is also the risk of ridicule, for example at the showing of 'Impression', critics described the show as "painting created in a state of delirium tremens" and the risk of hatred, for example Marcus Harvey's 'Myra' the giant reproduction of the newspaper image of Myra Hindley constructed with children's hand prints. Artists take many different kinds of risk. Creativity should be allowed to be 'risky' and artists have the training to facilitate these risks which can instigate and investigate unconventional perspectives. These 'risks' or 'experiments' could have the 
same impact on the game industry as 'Impression' did to the art world, raising the perception of players and allowing multiplicities of engagement with games. Opening up new possibilities of innovative self-interaction between computer games and their players, allows new opportunities to access different areas of narrative interaction, allowing connection to players' emotions to create alternative 'self-blendings'.

Tetsuya Mizuguchi, game developer, created the game "Rez", (Sonic Team Sega, 2001) [15] whose aesthetic levels were derived from the synaesthesia school of artists, painters, and composers, and the painterly aesthetic of Kandinsky.
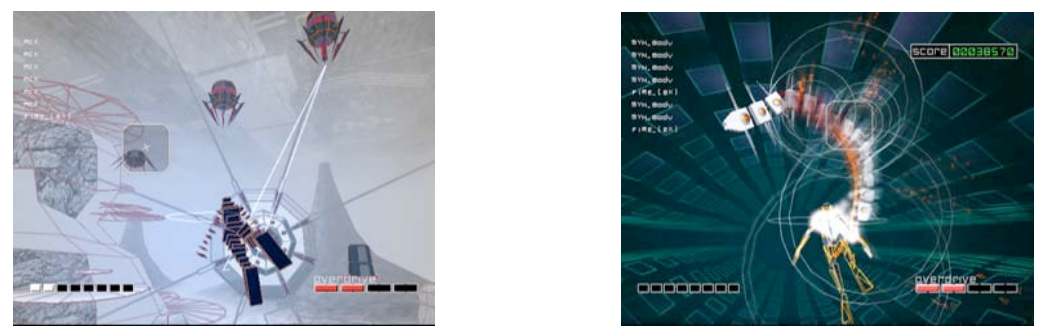

Figure 1. Rez images

When playing "Rez" the player is immersed into an abstract patterning of sounds, visual images, brilliant colours, and abstract designs which are linked to music as seen in Figure 1. Katamari Damacy, illustrated in Figures $2 \& 3$, is also another innovative game where the surreal concept is a real think-outside-the-box game genre.

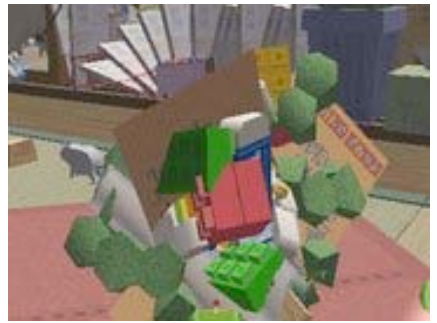

Figure 2. Katamari ‘clumps

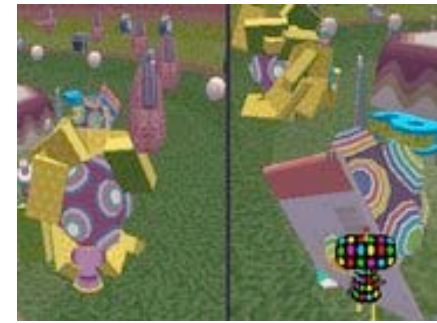

Figure 3. Katamari 'clumps'

When players start the game, they are given a katamari (which roughly translates to "clump" in Japanese) not much bigger than the player. "You roll this around, collecting anything you can to increase its size, (Davis, 2006) [2] There are certain constellations that require a katamari to be built out of specific stuff that corresponds to the shape of the constellation. For example, Pisces requires the player to collect a number of fish, while players need to find the biggest bear that they can to create Ursa Major. DaviesHe continued "[Players] go from "rolling along a tabletop to ravaging through city streets, picking up momentum and skyscrapers along the way." The disparity of the game of Katamari Damacy nevertheless has similarities with the artist Dew Harrison's 'StarGlass' a screen grab of part of which is shown in Figure 4 Originally intended as an interactive art piece, this 'art' or 'game' shows how user engagement was encouraged through means other than those usually employed in games. 


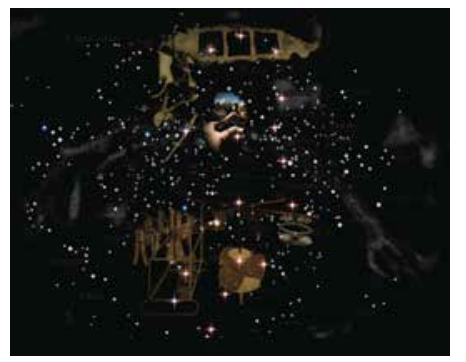

Figure 4. Virgoan stars (pinkish), the 3 smaller stars (bluish, on left) and a Duchampian object (Étant Donnés) Dew Harrison

Harrison argued "You could say that the aim of this computer game is to make sense of it through exploration, the strategy is the semantic association of the multimedia objects within it, as presented in the initial vision for the 'all-purpose machine' to augment human thinking - the computer." (Harrison \& Parker 2006) [4]

Another game in these alternative haute game models is 'Madrid'. This game was created in response to the September 11th attacks to New York and the USA in response to the futility of war and terrorism. 'Madrid' (Newsgaming.com, 2004) [9] is a form of digital meditation: the player is invited to brighten candles in memory of victims of terrorist attacks all over the world; the player has to re-light the candles as they go out. As the player finds it is impossible to get all the candles re-ignited for the whole of the game scene, it is a metaphor commenting on the futility of war as shown in Figures $5 \& 6$.

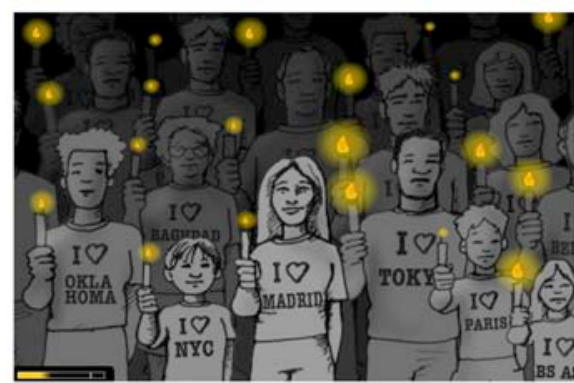

Figure 5. 'Madrid'

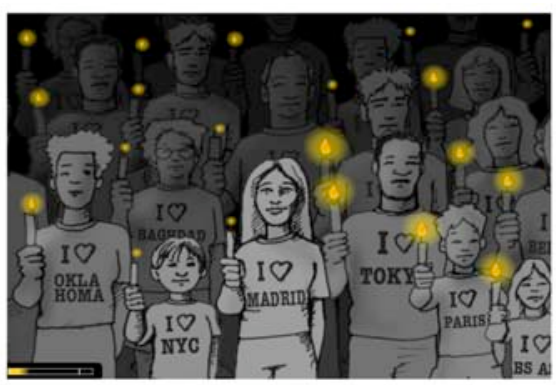

Figure 6. 'Madrid'

\section{HAUTE}

Haute means elegant or high, couture means dressmaking, sewing, or needlework and so the two combined imply excellent artistry, experimentation and investigation with the fashioning of garments. In this paper I argue that this is comparable to artists producing fine art pieces which investigate alternative interactions, for example Rachel Whiteread's use of cement to create art-pieces, instead of being used to construct buildings, or Jackson Pollock experimenting with paint in an abstract manner, or computer games producing alternative methods of interaction, immersion and blendings with players self and self-identity. Haute couture may be unattainable to the general clothes buyer, but-by 'selling a dream'-fashion shows attract huge media attention and gain enormous publicity for the couture houses. They are important to the rest of the industry for the innovative ideas its designers produce. Other firms may copy these designs and re-produce them at smaller cost to the buyer, but the ideas are created in the haute couture market. Similarly for Haute Cuisine 'super-chefs' are producing complicated food with lots of process which would not be created by a normal person, 
but the fact that chefs produce these dishes becomes disseminated into our cultural awareness, affording us the possibility of creating-maybe not the original dish-but a simpler version of it, for example Delia Smith's use of ready-made meals. These industries sell a dream of the intangible as does the game industry, a dream of entering into a fantasy land of 'Haute' levels. Haute games allow the dissemination of ideas throughout the game industry, and produce ideas and alternative games-games most game developers do not have the time, money or ideas to implement.

\section{STRANGE SPACES}

Haute games created by artists set up situations to evoke emotional responses from viewers, relating philosophy and theories, utilising and facilitating personal intuition and rigorous perception to create pieces of work. These theories are endorsed in the work and in the production of the art work by construction, response and relation. Artists open our eyes to new ways to see. For example, the impressionists created new ways to look at paint, Duchamp created connections to investigate 3 and 4-dimensions in space and the writings of Bachelard spanned science, poetry and human consciousness. These unconventional syntheses of perspectives recognise the creativity of the human mind. When playing computer games and concentrating solely on the game play, are we losing part of our sense of self and blending with the game narrative, becoming 'another self'? Placing ourselves within the game space and combining with the game as we do when viewing a piece of art work, we become immersed in the object and surrender part of our self-identity to the object, inhabiting the internal self which the artist projects from their internal 'strange space' into the external world as shown in Figure 7.

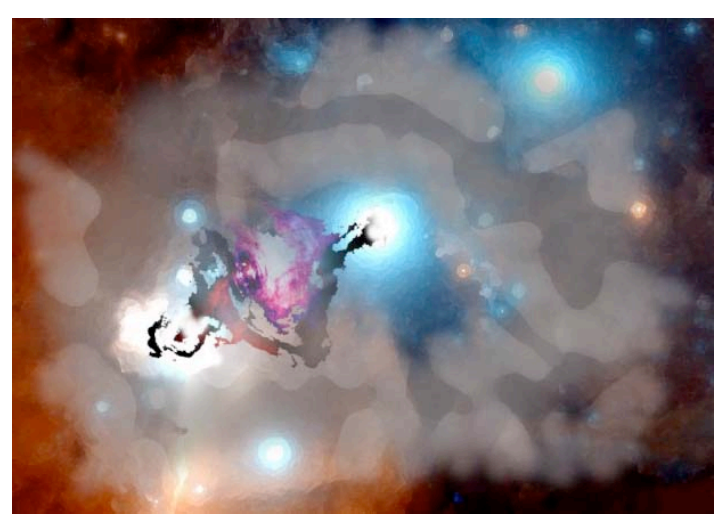

Figure 7. 'Strange Space’ digital image Maggie Parker 2007

\section{Game Modding}

What can happen when artists are given software tools and packages to 'play' with, play here meaning experimentation with models and materials? Artists can subvert software by 'modding' game levels, exploring and manipulating and blending different elements of the game play shell. They can explore materials, the artist changing a functional or aesthetic element in an existing game shell or in existing coding, creating their own version of how that coding will be displayed to create their own versions of 'games' to play as shown in Figure 8. JoDi's SOD is a modification of the game Wolfenstein. You can actually attempt to play the game, but everything is scrambled. 


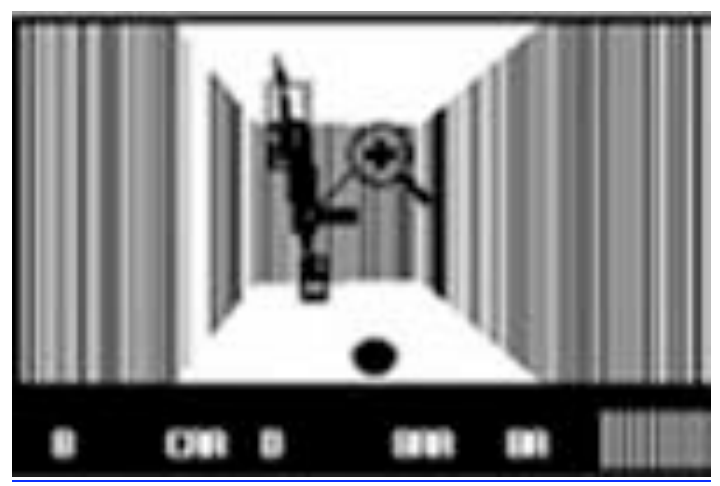

Figure 8. JoDI:SOD

\section{STAR WORLD}

If games are being connected by the popular media with violence, could they also not be connected with a gentler engagement with game play? Playing a computer game which blends with players' emotions to engage and interact in a gentle, protective and peaceful environment scenario could become as commonplace as playing beat 'em up', shoot 'em up games. Building on this history of genre-breaking haute games, I assembled an interactive computer game environment, Star World. This was developed to begin both an artistic and empirical investigation to create an alternative space in order to investigate whether creating a seductive, non-threatening environment would induce players to engage in an abstract space with abstract objects creating their own narrative?

\section{SYSTEM DESIGN}

In this 'veduta ideata', Star World became an idealised view based on an actual situation by adding entirely fictive elements and landscapes and illustrate 'ideal' spaces as practiced by architects such as Andrea Palladio (1570). [11] The environment consists of a realistically conceived scene that contains wholly imaginary elements. Star maps are textured onto a displacement-mapped twig landscape, producing stars wrapped around the bark of trees. Handmade paper scanned in and reproduced digitally becomes a rotating inner sphere, a real-world item transformed into a mythical landscape. Slow-moving animations create a hypnotic feast for the eyes; the stuff in the digital landscape may not exist, but the spirit of the artefacts exists in the space. All real-world objects have a surface texture, which is the surface roughness-not the surface colour or pattern which is especially important for close up shots. In a real world space this is an expected reaction to surface texture, however, in Star World; textures are placed in unexpected configurations and not re-created as real world textures as shown in Figure 9.
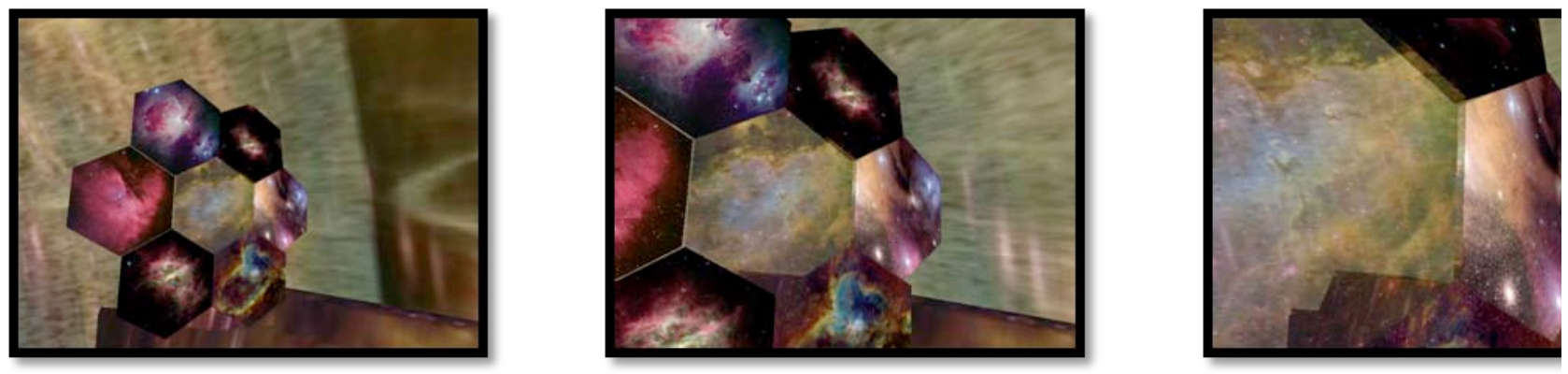

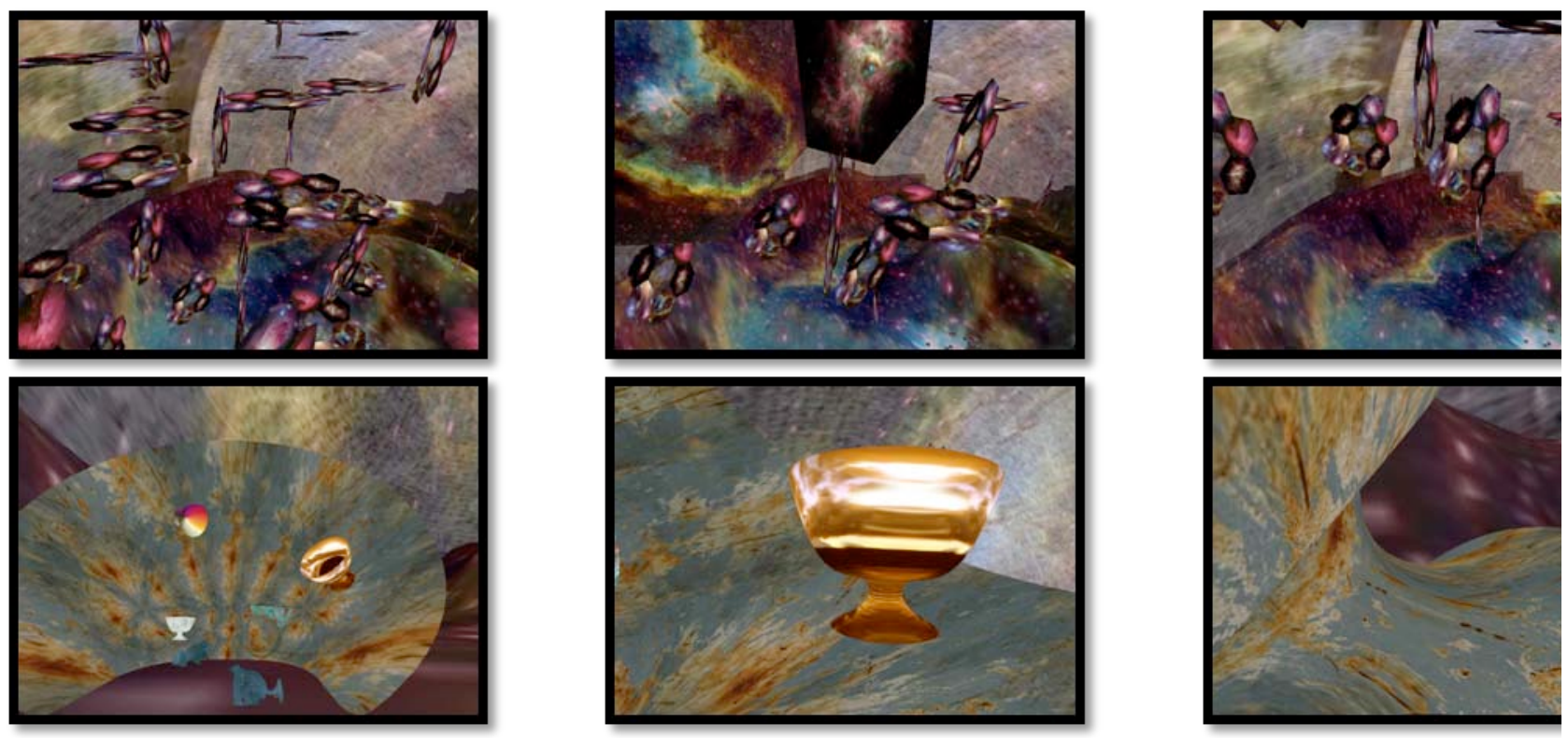

Figure 9. 'Star World' environment-game screenshots

This environment-or-game was exhibited in art spaces, conferences, and computer exhibitions. I observed viewers engaging with the piece seemed to dissolve, engage and 'blend' with environment once they realised there was nothing threatening to encounter and defeat. They enjoyed the time to 'chill-out' in a non-threatening environment. Viewers/players were much more engaged and not in an alert state as computer gamers naturally are in order to shoot anything that threatens them or to deal with a situation initiated via game-play to keep them 'alert and ready to defend'. (Eladahri, 2004) [3] Some viewers/players also reported that even though they had not had much experience in navigating computer games, they quickly mastered the controls and were able to navigate around the environment with the minimum of training. Easier methods of navigating a computer game environment created within navigators of the environment, a confidence in their skill level. Participants reported "I was really scared of computer games before, but if your environment is where I could begin to learn to play them then I would play more games". (Parker, 2005) [12]

\section{Responses to Star World}

As well as an art piece, Star World became an empirical test-bed. Participants were invited to navigate Star World and describe their experiences. They were asked to imagine how they would describe Star World to someone who had never navigated the environment and to remember what they felt, this retrospective 'think-aloud' teach-back (van de Veer, 1990) [17] generated protocols for the exploration of Star World. The protocols were transcribed and examined to see if themes could be identified. Each transcript was analysed in terms of themes which emerged from the spoken words of the participants as shown in Table 1.

$\begin{array}{lcc}\text { Themes } & \begin{array}{c}\text { Number } \\ \text { Themes }\end{array} \\ \text { Movement } & 471 \\ \text { Emotions } & 265 \\ \text { Environment } & 210\end{array}$




\begin{tabular}{ll} 
Abstract Objects & 164 \\
Verbs & 117 \\
Colours & 84 \\
Abstract & 67 \\
Concepts & 61 \\
Sounds & 24 \\
Relaxation & \\
\hline Table 1. Themes from teach-back task
\end{tabular}

The highest word count (471 words) related to movement. This can be related to this author's observation that, when observing players, game players wanted to 'run' everywhere in a game, thus creating 'exploration at super speed.' Even just watching game players play at this speed was not relaxing at all, so this author wanted movement through Star World to be slow and relaxing with no 'gamers' run' allowed, all movement taking place at a gentle 'waft and flow' throughout the navigation. (Csikszentmihalyi, 1996) [1] The quantity of words relating to movement seems to suggest the environment was built in such a way as to be a pleasant place to engage with. "As Ramachandran (2003) [14] hypothesised, "Every partial glimpse has to be pleasing enough to prompt further visual search, in other words the wiring of your visual centres to your emotional centres ensures that the very act of searching for the solution is pleasing."

\section{EMOTIONS}

Participants' emotions (265 mentions recorded) ranged through both positive and negative, for example from annoyance ( 7 recorded) to enjoyment (12 recorded). Participant 1 said they were most frustrated with the actual navigation controls which were the mouse and cursor key controls. "I was annoyed because I didn't get anywheregot to get somewhere". Only two participants reported feeling frustrated with the actual environment. Both participants who mentioned this were game-players and the author hypothesises game-players were used to a surge in adrenalin and did not get the 'rush' or 'buzz' they connected with game playing as the controls to navigate were set at a steady speed and could not be adjusted. Therefore, they became frustrated with this lack of control.

\section{Abstract ObJects}

Participants identified in various ways the hexagon objects placed in the environment, naming them as hexagons, birds, geese, space ships, fifty-pence pieces and others. In total the hexagons had 29 differing descriptions. "Looked like a woman sitting or a statuette sitting on a seashore" "I thought the birds were flying in a flock like jet rangers to blast you out of the sky but there was no interaction" "I saw the goblets.....I wanted to explore them". "Probing the willing suspension of self-identity and the willing suspension of disbelief is leading to experimenting within the aware and the unaware, presence and immersion happenings taking place between cyberspace and the 'real time' person inhabiting the space or operating the equipment". (Parker, et al., 2005) [13] Participants were unaware of what the abstract shapes actually were and as such 'named' them. Creating a narrative with objects, bringing them into a field of expertise and knowing and naming the object created within the viewer a power to 
believe they could give their opinion, achieve ownership and become the temporary possessors of the object.

\section{BLENDING}

One of the many interesting narratives [or codes] generated by participants was their interpretation of the abstract shapes "I reached the duck or geese shapes in the sky" although there were no such shapes in the environment or any shapes intended to represent these concepts. These results of the description of abstract shapes were usually in line with a participant's interests. This participant did describe her hobby as bird-watching. Another participant, who interpreted the shapes as "seaweed, shells and tadpoles", went for long walks on the beach to relax, and named the abstract shapes according to objects that were familiar to her. This relation to each participant's hobbies is significant, not in a scientific logical measurable method, but in identifying, engaging and transforming abstract shapes into something each participant could rationalise to create a personal, emotional, self-blend and connection. Abstract objects will often have connotations or meanings which come from within our own culture and society. These can sometimes be recognised consciously, but at other times are only apparent when we look for them. Artists are constantly engaging with and noticing these connotations, and cracking these cultural codes becomes part of art practice. Because Star World was constructed using abstract pieces, these pieces became attractors, these in turn activated participants strange space to project onto these abstract pieces their own personal schema and could interpret the imagery in a personal way, allowing a blending and engagement with a virtual environment in a way previously unexplored. To win and solve puzzles is normally the reason to play many games. However "There is nothing about the microprocessor or the monitor that requires games to be about shooting aliens or searching for treasure" (Adams, 2001), unexplored by many developers are the many possibilities that games can have to allow alternative outcomes to a game. Stafford, (2007) [16] argues for a new understanding of images, not simply as products of mental operations but as "constitutive cognitive processes". She discusses the puzzle of "binding", the process this author has termed "blending" which we both argue "make visible the invisible ordering of the human consciousness". In calling for art, science, philosophy, and technology to become a single investigation, she is asking for what some artists do as a matter of course when pursuing their work.

\section{Relaxation}

One of the intentions of creating Star World was to investigate the possibility of using virtual environments and computer-games to help alleviate stress, one of the reasons for loss of work time and heart disease (North, 2008). [10] Three participants thought the animation of the hexagons and the animation of the inside sphere was relaxing, 8 participants thought sound added to the notion of relaxation. One participant thought "the sound of the sea" helped to relax them. Nine participants thought movement was relaxing. One participant thought the experience was "pretty....and relaxing", another participant said they enjoyed "just sitting here watching...very relaxing". One participant thought the colours were relaxing, "colours...calming sound...relaxing...quite enjoyed it". This participant also observed that "just sitting here watching...very relaxing". Another participant said "I was uneasy with the floating goblets, why are they floating in the sky-but I didn't feel threatened at all and now I feel relaxed." Two participants thought they did not think they were relaxed and 
said they "Don't feel relaxed but was not relaxed before". Another participant said "gloomy depressing colours made me tired, not relaxing". Overall, when navigating Star World, relaxation was mentioned 24 times by 23 out of the 28 participants in the study, and when shown in alternative art spaces, for example the Department for Fundamental Physics at the University of Durham, people who navigated the environment mentioned similar experiences. Harrison \& Parker explained Star World was intended as a stress free game environment for players who want to relax in their leisure time. This game uses visual aesthetics, often abstract and painterly, it extends game design into something that could easily be situated in an art space and begs the question "Why aren't creative games shown in white cube galleries?"

\section{Conclusion}

Game environments offer an alternative way of 'viewing' images contained within a monitor or TV screen. Works of art are cognitive devices aimed at the production of rich cognitive effects. Thus it can be argued, in the light of what is known about human cognition, that aesthetic experience is a by-product of the exercise of more fundamental cognitive faculties such as perception, imagination, and returning to the introduction in this paper-love and seduction. Works of art are never grasped directly. Rather, in an aesthetic experience, a subject directly perceives a certain object or event (a canvas, a display of pixels, a series of sounds), and this perception gives rise to a cognitive activity of a special, aesthetic type. Abstract shapes were included in Star World and these visual clues became maps onto which participants mapped their own self-identity and narrative which allowed participants to blend with an abstract illogical world. As discussed by Lackoff \& Johnson (1980 \& 1987) [7] [8] these abstract objects required participants to engage and apply their personal image schemata causing them to make connections, or blendings with to what they were viewing. This interaction took place in and throughout the time of navigation of Star World. As participants navigated the environment, they created "multi-modal patterns of experience, not simply visual". If, as he argues, "all our symbolic expression and interaction are tied intimately to our embodiment and to the pervasive aesthetic characteristics of all experience", then Star World tapped into that multi-modal experience. For example the experience of one participant "I reached the duck or geese shapes in the sky" when her hobby was bird watching. This seems to support Johnson's argument (1999) [6] that "aesthetic aspects of experience structure every dimension of our experience and understanding". More generally, players' positive response to their experience, whether in terms of flow (Hsu $\& \mathrm{Lu}, 2004$ ) [5] or otherwise, can lead to a higher acceptance of a game and ultimately higher success in the market of games. One important contribution of this investigation has highlighted the need for alternative computer-game designs, computer-games which relate and respond to different player-emotions and types. Another important contribution was the integration-or-blending of dissimilar fields and practices which prove a synthesis can be achieved, and methods and practices can be used to create alternative processes.

\section{References}

[1] CSIKSZENTMIHALYI, M., (1996). "Creativity: Flow and the Psychology of Discovery and Invention” New York: Harper Perennial. 
[2] DAVIS, R., (2006). "Katamari Damarcy" Available at http://uk.gamespot.com/ps2/puzzle/katamaridamashii/review.html Posted Sep 21, 2004 1:29 am GMT [accessed 17 July 2006]

[3] ELADHARI, M., (2004). "Personal Observation" University of Teesside.

[4] HARRISON, D., , M., (2006). "Game-like art/Art-like game: Reflections of a Star World in a StarGlass" In proceedings at Media Terra Conference Athens.

[5] HSU, C.L., Lu, H.P., (2003). "Why do people play on-line games? An extended TAM with social influences and flow experience" Information \& Management 41(7): 853-868.

[6] JOHNSON, M. (1987). "The Body in the Mind: The Bodily Basis of Meaning, Imagination, and Reason" University of Chicago

LAKOFF, G, \& JOHNSON, M., (1980). “Metaphors We Live By.” Chicago: University of Chicago Press

LAKOFF, G, \& JOHNSON, M., (1999). "Philosophy in the Flesh: The Embodied Mind and Its Challenge to Western Thought" Basic Books, 1999

Newsgaming.com (2004) Newsgaming.com designs video games that are also tools for better understanding the world Available at http://www.newsgaming.com [accessed 22 September 2004]

NORTH, E., (2008) "Stress Link to Heart Disease Video link Available at http://search.bbc.co.uk/cgi-

$\mathrm{bin} / \mathrm{search} /$ results.pl?go=homepage \&scope=all\&tab=all\&q=stress+in+the+wor $\mathrm{k}+$ place $\&$ Search $=$ Search [accessed 24 January 2008]

[11] PALLADIO, A., (1570). "The Four Books of Architecture"

[12] PARKER, M., (2005). "Digital City Fellows exhibition" Senior Fellow Digital City University of Teesside, Centre for Enterprise Reported by Andrew Burtenshaw

[13] PARKER, M., et al., (2005). "Game with a Purpose" In proceedings of Computer Graphics, Imaging \& Visualisation Conference, Institute of Automation, Beijing, China.

[14] RAMACHANDRAN, V., (2003). "The Artful Brain" Lecture 3 Available at http://www.bbc.co.uk/radio4/reith2003/lecture3.shtml [accessed 23 November 2003]

[15] Sonic Team, Sega., (2001). "Rez"Available at http://www.sonicteam.com/rez/e/game/index.html [accessed 3 May 2005]

[16] STAFFORD, B., (2007). "Echo Objects, The cognitive Work of Images" University Of Chicago Press

[17] VAN DER VEER, G. C., WHITE. T. N., (1990). "University education on human-computer interaction: The Dutch situation" INTERACT 1990: Cambridge, UK. 